BLS 35, No 1 2009. DOI: http://dx.doi.org/10.3765/bls.v35i1.3627

(published by the Berkeley Linguistics Society and the Linguistic Society of America)

\title{
NPIs Pragmatically*
}

\author{
ELENA HERBURGER and SIMON MAUCK \\ Georgetown University and Maagha Press
}

\section{The Licensing Question}

Following Ladusaw (1980), NPIs are (widely) held to be licensed in downward entailing (DE) contexts:
a. I do*(n't) see anyone.
b. Every/*some student(s) with any sense asked for help.
c. If you ever see a copy of this book, get it for me, please.
d. I doubt/*believe that anyone really knows how things will be next summer.

Some contexts are more obviously downward entailing than others. Thus, it is clear that the negation in (1a) creates a DE context, but it is not quite as clear that the if in (1c) does. Moreover, some NPIs only like certain kinds of DE contexts and which ones occur in which contexts is an interesting question which has received considerable attention in the literature. We would like to abstract away from these issues here, however, and address another one: Why should NPIs be limited to semantically negative contexts, contexts that invert the direction of entailment (Fauconnier 1975)? Recent analyses attempt to answer this question by exploiting the semantics of NPIs (cf. Krifka 1995, Lahiri 1998, Chierchia 2006). We argue that while the distributional restrictions of NPIs are indeed related to their meanings, the distributional restriction of NPIs cannot be derived from for their meanings.

\section{A Semantic Answer to the Licensing Question?}

Instead of describing the details of the various analyses which try to answer the Licensing Question by exploiting the semantics of NPI, we focus on the basic idea underlying these accounts (For some details see the appendix):

\footnotetext{
* The authors would like to thank the audience at the Berkeley Linguistics Society meeting for their comments and questions.
} 


\section{Elena Herburger and Simon Mauck}

(2) Semantic answer to the Licensing Question:

NPIs are low scalar elements that come with an operator requiring them to be the semantically strongest alternative.

By low scalar elements we mean those elements that occupy the bottom rung on a Horn scale (Horn 1972). For instance, some is the bottom element of a scale of quantifiers including many, most and all. In non-negative contexts, some is the weakest of these scalar alternatives (i.e. a sentence with many entails an alternative with some). But in negative contexts, some is the strongest of the alternatives, as negation inverts the direction of entailment (Fauconnier 1975). Since low scalar elements are semantically strong in precisely those contexts that are DE, then, if one assumes (2), it follows that NPIs are limited to DE contexts.

Several versions of (2) involve the presence of some form of even, either a concrete one (Lahiri 1998, see also Lee and Horn 1994) or an abstract one (e.g. Chierchia 2006). To sketch the workings of the semantic answer to the Licensing Question, it is thus useful to first look at even.

The analyses mentioned above assume, with Kartunnen and Peters (1979), that 'even' picks out the least likely of the alternatives. (3), for instance, is said to give rise to the inferences in (4), which in Kartunnen and Peters's account are phrased as conventional implicatures:

(3) Even DEBBIE thought they had gone too far.

a. Conventional scalar implicature:

Debbie was the least likely among the relevant people to think that they had gone too far.

b. Existential implicature:

Someone other than Debbie thought that they had gone too far.

There is a relationship between likelihood and entailment or semantic strength for scalar terms: If a sentence A that employs a scalar term is less likely than a sentence $\mathrm{B}$, where $\mathrm{B}$ employs an alternative on the same scale, then the truth of $\mathrm{A}$ guarantees the truth $\mathrm{B}$, i.e. A entails/is semantically stronger than $\mathrm{B}$. This means that if NPIs are composed of 'even' and low scalar elements, as (2) hypothesizes, then the relevant low scalar element has to offer the least likely or semantically strongest alternative. Since this is only the case in DE contexts, it follows that even + a low scalar element is restricted to these contexts. When applied to our initial example in (1a) this analysis works as follows:

$$
\text { I don’t see anyone. (=1a) }
$$

The sentence implicates that for the speaker to not see one person is less likely than for him or her not to see several people. This makes sense because not seeing one person entails not seeing several. By the same token, if the negation is absent, 


\section{NPIs Pragmatically}

the implicature generated by even and the entailment properties of the sentence clash; for the speaker to see one person is not less likely than for him or her to see several people, but rather more likely because whenever you see two or more persons, you also see one.

Despite its elegance, the semantic answer to the licensing questions faces a number of difficulties (Herburger and Mauck 2007, Herburger to appear). Two, relatively minor ones concern even. Even though some languages use an overt even as part of their NPIs (e.g. Hindi), there are many NPIs which lack a visible instance of even, for example any and ever. For these, a tacit even (feature) has to be posited (cf. Chierchia 2006). Also, even may not pick out the least likely of the alternatives, but the most informative one (Kay 1990, Herburger 2000). If so, an operator other than even must actually be posited to pick out the semantically strongest alternative (cf. Chierchia's 2006 and Krifka's 1995 abstract operators). This somewhat weakens the appeal to cases where NPIs contain an overt form of even.

There are also four more serious problems. First, for the even analyses to work, it has to be stipulated that the licensing operator takes scope over the element responsible for the DE context, no matter how far away that element is. Second, unlicensed NPIs actually seem to result in ungrammaticality rather than pragmatic infelicity, contrary to what these analyses would predict (see also Gianniakidou 2001). Third, note that the presence of the licensing operator does not follow from the low scalar nature of NPIs - not all low scalar expressions are NPIs. This, however, means that the licensing operator has to be effectively stipulated. Fourth, a number of NPIs are not low scalar elements, e.g. much (in its NPI usage) and Spanish gran cosa. (Israel 1996). The even analyses do not extend to these in any obvious way.

a. I do*(n't) have much time.

b. La película no fue gran cosa. The film not was big thing 'The film wasn't good.'

Given these issues, it may be worthwhile to contemplate an alternative to the semantic answer to the licensing question in (2). In what follows, we argue that it is not the semantics of NPIs that directly accounts for their distribution, but ultimately the presence of a lexical feature. The presence of this feature relates to the pragmatic usefulness of these expressions in certain contexts.

\section{On the Pragmatics of NPIs}

The pragmatics of NPIs and the role it might play in their licensing has not gone unnoticed. In particular, Israel (1996, 1997) develops an analysis of NPI licensing that does not only recognize that not all NPIs are low scalar elements but also pays close attention to how they function in discourse. We take his analysis as a point of departure. 


\section{Elena Herburger and Simon Mauck}

\subsection{Israel's (1996) Q- and I-Values}

Israel (1996) points to the heterogeneity of NPIs and accounts for the licensing of two classes of NPIs, namely low scalar NPIs like any and relatively high scalar NPIs like much, by positing two values, a q-value and an i-value. The q-value encodes the rung of the NPI on a Horn scale (Horn 1972); it is high if the NPI is high on the scale and low if it is low. The i-value, in turn, signals emphasis and semantic strength. A high i-value means that the 'text proposition' (TP; the sentence containing the NPI) entails an alternative 'context proposition' (CP), where the CP involves a mid-scalar expression (the 'scalar norm'). A low i-value does the opposite. It encodes understatement and requires the TP to be entailed by the CP. Using these values, Israel proposes to lexically classify the two kinds of NPIs he discerns in the following way:

$$
\begin{aligned}
& \text { a. low q-value }+ \text { high i-value }=\text { low scalar NPIs (any, ever, } \\
& \text { budge an inch) }
\end{aligned}
$$

b. high q-value + low i-value $=$ high scalar NPIs (much, gran cosa, long)

The central point is that the classification in (7) predicts that NPIs will only appear in DE contexts. To see why this is so, let us first consider the low scalar NPIs described in (7a). A TP in which a low scalar element appears will only entail a $\mathrm{CP}$ involving a mid scalar element if both are negative:

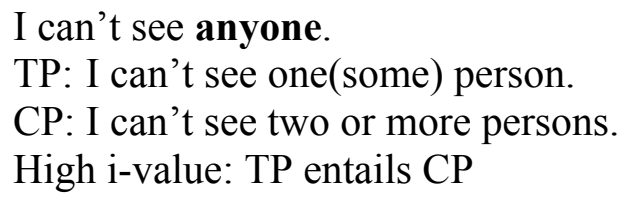

Turning now to the high scalar NPIs described in (7b), note that a TP with a high scalar element will only be entailed by a CP involving a mid scalar element, as demanded by the high i-value, if both TP and $\mathrm{CP}$ are negative:

(9) I don't have much time.

TP: I don't have a large amount of time.

CP: I don't have a medium amount of time.

Low i-value: CP entails TP

As appealing as this account is, it, too, raises some issues. First, what is the scalar norm? Sometimes what is at the bottom of the scale may in fact be what is 'normal,' but Israel is forced to appeal to a CP that indicates the middle of a scale, no matter what 'normal' in a given context may be. Second, all low scalar elements, regardless of whether they are NPIs are not, lead to semantically strong and hence potentially emphatic claims in DE contexts. Likewise, all high scalar expressions lend themselves to semantically weak and hence potentially understating claims in 


\section{NPIs Pragmatically}

DE contexts. This just follows from the nature of scales and the semantics of negation.
a. I can't see one (ONE) person.
b. I can't see two people.
c. One person has to volunteer to drive him back to the airport.

(10a) entails (10b), showing that one is semantically stronger than two, but (10c) shows that it is not an NPI. If, then, emphasis or the lack thereof follows directly from the scalar rung, the i-value does not really mark (lack of) emphasis, despite its name, but ultimately marks (in combination with the 'right' q-value) that the element in question is an NPI. Might we then not call things by their name and say that NPIs are lexically marked as NPIs?

\subsection{The Pragmatic Road to Formal NPI-hood}

The discussion of both the semantic licensing accounts and Israel's more pragmatically flavored account lead us to the following conclusion regarding the Licensing Question (Herburger and Mauck 2007, Herburger to appear):

(11) A syntactic answer to the Licensing Question:

An NPI differs from a non-NPI in carrying a formal feature. For concreteness, let us call it [u-neg].

While we believe that NPI licensing is a syntactic phenomenon, there is clearly a relationship between scales and NPIs. The rung an NPI occupies on a scale does not derive its distribution, but it 'merely' has pragmatic consequences that help explain how the distribution could come about.

Beginning with low scalar NPIs, we saw earlier that low scalar elements in negative contexts are semantically strong. This makes them pragmatically useful when one wants to make a strong or emphatic claim (Jespersen 1917). We believe that this pragmatic usefulness can lead some low scalar elements to being lexically restricted to negative contexts. Which ones are, however, is to some extent arbitrary. Consider in this context for instance the difference between any and some; both arguably denote existential quantifiers and occupy the bottom rung of a quantity scale (assuming that any is a low scalar element, cf. Carlson 1980, and also the appendix), but only any is an NPI.

As for relatively high scalar elements, they are pragmatically valuable in $\mathrm{DE}$ contexts in a different way: they allow for a rather weak, evasive claim and, as a consequence, they also allow for understatement. (12) thus can be taken to mean that the speaker has some but not much time (weak claim) or that she has no time at all (understatement):

(12) I don't have much time. 


\section{Elena Herburger and Simon Mauck}

The weak claim follows from a regular scalar implicature (Levinson 2000): Quantity implicates the negation of stronger alternatives. Since negation reverses the direction of entailment, the stronger alternative to much in (12) is some, i.e. (12) implicates that it is not the case that the speaker does not have some time, which amounts to saying that she has some time. The understated claim, we argue, arises when Quantity is suspended, and the speaker did not say everything there was to say. Since Quantity is inoperative, no scalar implicature is generated and (12) does not implicate the negation of 'I don't have some time'. In sum, we both expressions that lead to strong claims in negative contexts (low scalar expressions) and those that lend themselves to understated claims are pragmatically useful. Some, because of this, become restricted to such contexts. Those that do, bear a feature marking them as NPIs ([u-neg]).

\subsection{The Relative Arbitrariness of NPI-hood}

On our view, any expression with the 'right' scalar value can in principle be an NPI but does not have to. Because being an NPI amounts to nothing more than bearing a feature, we expect that over time expressions might change their status. Thus, it would not be surprising if the same element could be an NPI at one point in time and not in another. There may indeed be evidence for this, as we would like to show now.

According to Jäger (2006, 2007), the existential German einig- 'some', which can nowadays freely occur in non-DE contexts, used to be an NPI in Old High German.

(13) buuzssan einigan zuuiuun ist dhanne archennit, dhazs

Without any doubt is then recognized that 'It is recognized without any doubt that...' (OHG; Isidor VI.5)

The fact that in Modern High German, einig- still has an existential meaning but no longer the distribution of an NPIs suggests, in our terms, that einig- used to have a [u-neg] feature, but no longer does.

Similarly, Greek kanenas 'anyone' derives from kai 'even' and henas 'one'. Strikingly, while it is employed in many dialects of Modern Greek as a typical even NPI, it is also used as a simple, positive indefinite in some dialects, notably Cretan (Kiparsky and Condoravdi 2006). For us this means that in Cretan kianenas does not bear a [u-neg] feature. (Note that it also supports the claim that it is not the even in even NPIs that is responsible for their distribution.)

$$
\begin{aligned}
& \text { kianenas perastikos da perase ki èkopse ta portokalia } \\
& \text { some passerby here passed-by and cut the oranges }
\end{aligned}
$$

In the two examples we have discussed so far, a lexical item that has the NPI feature [u-neg] loses it at some point in its history. One might also wonder if it is possible for [u-neg] to become [i-neg], that is if it is possible for an uninter- 


\section{NPIs Pragmatically}

pretable feature, which simply demands the presence of a negative element elsewhere, to change into an interpretable feature, which makes a clear semantic contribution of its own namely negation. We think that this may indeed be possible. More specifically, we think it offers a way of conceptualizing part of the Jespersen Cycle, where an NPI over the course of time comes to be negative element in its own right. For instance, nobody, which used to function as an NPI in Middle English and still does so in many dialects, became a negative element in many dialects of early Modern English and is used this way today in standard English dialects. In other words, depending on the dialect/era, (1a) (I didn't see anyone) can be expressed by either (15a) or (15b):

$$
\begin{array}{ll}
\text { a. I didn't see nobody. } & \text { dialectal, historical } \\
\text { b. I saw nobody. } & \text { standard }
\end{array}
$$

If what we argue in this paper is correct, the fact that nobody was historically an NPI and is now (standardly) a negative quantifier amounts to saying that nobody historically bore a [u-neg] feature but now bears an [i-neg] feature.

Interestingly, the Jespersen Cycle also seems to be taking place in at least one language that has even NPIs, namely Modern Hebrew, which has morphologically complex NPIs containing an instance of even (af). ${ }^{1}$ Though these NPIs generally require negation to be licensed, this is not the case in elliptical answers, as shown in (16). This might suggest that these elements are on their way to becoming negative expressions in their own right, similar to English nobody.

$$
\begin{aligned}
& \text { A: mi ba? } \\
& \text { who came } \\
& \text { 'Who came?' } \\
& \text { B: af exad }\left({ }^{*} 10\right) \\
& \text { even one not } \\
& \text { 'No one.' }
\end{aligned}
$$

What further lends credence to this suspicion is the fact that in the speech of some children and teenagers, af exad is starting to be used without negation in preverbal positions, even though it still requires a negation when it appears in a postverbal position:

$$
\begin{aligned}
& \text { af exad oxel et ha-uga } \\
& \text { even one eats acc the-cake } \\
& \text { 'No one is eating the cake' }
\end{aligned}
$$

\footnotetext{
${ }^{1}$ While afilu (literally af 'even' + ilu 'counterfactual conditional') has replaced af as 'even' in standard oral Hebrew, of is still used in high register and newspaper Hebrew, and can be understood as meaning 'even' by those who learn these varieties of Hebrew (which are taught in school).
} 


\section{Elena Herburger and Simon Mauck}

This change would seem to be unexpected if the distribution of NPIs depended on even, but can be understood if, as we have proposed, NPIs are somewhat arbitrarily marked by a syntactic feature that can change over time. In particular, we can surmise that currently even expressions of the relevant sort in Hebrew are ambiguous at this point and marked either [u-neg], in which case they function like NPIs, or [i-neg], in which case they are negative. A similar account can be extended to n-words in languages like Spanish if they are ambiguous between NPIs and genuinely negative expressions (Herburger 2001).

\section{Conclusion and an Open Question}

We have argued in this paper that what makes a particular item with the requisite scalar (and hence pragmatic) disposition an NPI is 'merely' a formal feature, [uneg] and that NPI licensing ultimately amounts to a process of feature checking. We also speculated that expressions of the right scalar sort cannot only acquire this feature in the course of history, they can also lose it. Moreover, the feature can acquire semantic content and turn into [i-neg]. This offers a way of conceptualizing part of the Jespersen Cycle. If NPI licensing amounts to feature checking, this of course raises the question of how this feature checking works. One possible answer might involve substantive decomposition of NPI licensors into [neg] + something else, cf. Ludlow's L* (Herburger and Mauck 2007). We are currently exploring whether and how this would offer an account of the syntactic licensing of NPIs.

\section{Appendix: Some Semantic Licensing Accounts}

In this section we very briefly summarize some of the even accounts mentioned earlier in the paper and some of their precursors. Our discussion is meant to illustrative rather than exhaustive.

\subsection{Kadmon and Landman (1993)}

While Kadmon and Landman's (1993) account does not posit that NPIs contain an even or trigger the presence of an even-like operator, it does use the internal semantics of NPIs to explain their distribution and would seem to have inspired many of the proposals that followed it. According to Kadmon and Landman, any, on both its NPI and free choice interpretation (the latter of which we will not discuss) is an indefinite, like some, but with added features of 'widening' and 'strengthening'. The widening applies to the domain of quantification of the noun the NPI modifies. For instance, according to Kadmon and Landman, some potatoes might normally rule out very small potatoes or moldy potatoes, but any potatoes includes in its domain of quantification all potatoes, including the marginal ones. The strengthening induced by any then requires that the widening it induces be a stronger statement, i.e. that the statement on the widened interpretation will entail the statement on the normal, narrow interpretation (much as even requires the sentence it appears in to entail a weaker alternative). This will happen only in downward entailing environments. To see this, consider: 


\section{NPIs Pragmatically}

I don't have any potatoes.

If any induces widening, (18) states that the speaker does not have potatoes, normal ones or very small ones. This entails a statement without the widening, that the speaker does not have normal potatoes. Now consider a sentence without negation:

*I have any potatoes.

If the same widening is involved, the requirement for strengthening is not satisfied, because the wide meaning of 'I have potatoes of some kind (normal or very small)' does not entail the narrow meaning of 'I have normal potatoes'. Thus, the NPI is excluded. Of course, much on this account depends on any having a larger quantificational domain than some. Several authors have voiced skepticism (Krifka 1995, Lahiri 1998), pointing out that only stressed any seems to induce something that can be called widening, whereas 'normal' unstressed any seems to have a quantificational domain similar to that of some.

\subsection{Lee and Horn (1994)}

Lee and Horn (1994) claim that the distribution of any results from the fact that it is an indefinite with an incorporated even, where even has the meaning described in section 1. Rather than making use of the implicatures that even generates, however, Lee and Horn make use of the fact that these implicatures require the existence of a scale of likelihood associated with the sentence. They then posit that any is licensed only where such a scale can be constructed. Whether or not a scale can be constructed can be determined by whether or not even a single or even + a superlative can appear in a sentence. Thus, pointing to examples like (20) and (21), Lee and Horn predict that any can occur in a sentence if even a single or even + a superlative can occur in the same position in that sentence:

(20) a. *There is any boy running around in the garden.

b. *There is even a single boy running around in the garden.

(21) a. There isn't any boy running around in the garden.

b. There isn't even a single boy running around in the garden.

Lee and Horn also noted that many languages, such as Hindi, Modern Hebrew, Korean, and Turkish include an instance of even as part of their NPIs, a fact that would be important in some of the accounts of NPI licensing that followed theirs.

\subsection{Krifka (1995)}

Krifka (1995) assumes that NPIs are low scalar expressions that trigger alternatives. He furthermore assumes that the sentences they appear in are interpreted in terms of structured meanings where the NPI is 'foreground' and the rest of the 


\section{Elena Herburger and Simon Mauck}

clause is abstracted over and represents the 'background'. He then posits that the alternatives for 'weak' NPIs (e.g. any, ever) include more specific instances, e.g. books, pencils for anything. The alternatives for 'strong' NPIs (e.g. ANYthing, at all, a drop) are the same with the additional proviso that no marginal cases are included (cf. Kadmon and Landman 1993). He then posits that weak NPIs trigger the presence of Scal.Assert and strong NPIs that of Empahtic.Assert.

Scal.Assert is an operator that gives rise to an implicature, in particular the implicature that no alternatives to the weak NPI result in true assertions. On this account, a sentence like (22) is ruled out because of an implicature that conflicts with the meaning; if Mary saw something then it is true, rather than false, contrary to what the implicature triggered by Scal.Assert would demand, that she saw something specific, e.g. a pencil. (23), in contrast, is acceptable because the implicature generated by Scal.Assert is consistent with the meaning of the sentence:

*Mary saw anything.

Mary didn't see anything.

Like even, Emphatic.Assert requires that the proposition asserted be less likely than any alternative proposition and, furthermore requires that it also be less likely than the conjunction of all alternative proposition involving minor entities. The result is that the asserted proposition has to be extremely unlikely and that minor entities are excluded (a la Kadmon and Landman 1993).

\subsection{Lahiri (1998)}

Lahiri (1998) observes that in Hindi NPIs consist of a low scalar element + bhii 'even.'

$$
\begin{aligned}
& \text { koii bhii nahiiN aayaa } \\
& \text { some even not came } \\
& \text { 'No one came.' }
\end{aligned}
$$

He argues that bhii is a focus particle with a meaning similar to that of even and which picks out the least likely and hence semantically strongest of the focus alternatives. As we saw in section 1, this predicts that the relevant expressions are only felicitous in those contexts where low scalar elements are the strongest, namely DE contexts (Lahiri also extends his analysis to free choice context, but we will not go into that here.).

\subsection{Chierchia (2006)}

Finally, Chierchia (2006) maintains that the NPI any is an existential quantifier with a widened domain (cf. Kadmon and Landman 1993), which comes with alternative domains involving subsets of the original quantificational domain. He furthermore assumes that the occurrence of the NPI any triggers the presence of 


\section{NPIs Pragmatically}

an even-like operator $\mathrm{E}_{\mathrm{c}}$. $\mathrm{E}_{\mathrm{c}}$ requires that the proposition expressed by the sentence entail all alternative propositions derived with the help of the alternative domains (cf. Krifka's 1995 Emphatic.Assert, cf. even). The operator thus has the meaning in (25):

$$
\mathrm{E}_{\mathrm{c}}(\mathrm{p})=\mathrm{p} \wedge \forall \mathrm{q} \in \mathrm{C}[\mathrm{p} \subseteq \mathrm{q}]
$$

Since the alternative domains are smaller than the original, widened one, a proposition involving an element in the original domain will only entail a proposition involving an element from a smaller alternative domain if it is embedded in a DE context. This has the desired effect of restricting NPIs to DE contexts.

\section{References}

Carlson, Gregory. 1980. Polarity any is existential. Linguistic Inquiry 11:799-804.

Chierchia, Gennaro. 2006. Broaden your views: Implicatures of domain widening and the "logicality" of language. Linguistic Inquiry 37:535-590.

Fauconnier, Gilles. 1975. Pragmatic scales and logical structure. Linguistic Inquiry 6:353-376.

Giannakidou, Anastasia. 2001. The meaning of free choice. Linguistics and Philosophy 24:659-735.

Herburger, Elena. 2001. The negative concord puzzle revisited. Natural Language Semantics 9:289-333.

Herburger, Elena. to appear. Negation. In C. Maienborn, K. von Heusinger, and P. Portner, eds., Semantics: An international handbook of natural language meaning. Berlin: Mouton de Gruyter

Herburger, Elena, and Simon Mauck. 2007. A new look at Ladusaw's puzzle. In H. Zeijlstra and J.-P. Soehn, eds., Proceedings of the Workshop on Negation and Polarity, 64-71. University of Tübingen.

Horn, Laurence. 1972. On the semantic properties of the logical operators in English. Bloomington, IN: Indiana University Linguistics Club.

Israel, Michael. 1996. Polarity sensitively as lexical semantics. Linguistics and Philosophy 19:619-666.

Israel, Michael. 1997. The scalar model of polarity sensitivity: The case of aspectual operators. In D. Forget, P. Hirschbühler, F. Martinau and M.L. Rivero, eds., Negation: Syntax and semantics, 209-230. Amsterdam: John Benjamins.

Jäger, Agnes. 2006. History of German negation. Ph.D. diss., University of Jena.

Jäger, Agnes. 2007. On the diachrony of polarity types of indefinites. In H. Zeijlstra and J.-P. Soehn, eds., Proceedings of the Workshop on Negation and Polarity, 78-84. University of Tübingen.

Jespersen, Otto. 1917. Negation in English and other languages. Kopenhagen: Vienskabenes Selskap. 
Elena Herburger and Simon Mauck

Kadmon, Nirit, and Fred Landman. 1993. Any. Linguistics and Philosophy 16:353-422.

Kartunnen, Lauri, and Stanley Peters. 1979. Conventional implicature. In C.-Y. Oh and D. Dinneen, eds., Syntax and Semantics, Vol. 11: Presuppositions, 1-56. New York: Academic Press.

Kay, Paul. 1990. Even. Linguistics and Philosophy 13: 59-111.

Kiparsky, Paul, and Cleo Condoravdi. 2006. Tracking Jespersen's Cycles. In M. Janse, B.D. Joseph and A. Ralli, eds., Proceedings of the $2^{\text {nd }}$ International Conference of Modern Greek Dialects and Linguistic Theory. Mytilene: Doukas.

Krifka, Manfred 1995. The semantics and pragmatics of polarity items. Linguistic Analysis 25:209-257.

Ladusaw, William. 1980. Polarity sensitivity as inherent scope relations. New York: Garland.

Lahiri, Utpal. 1998. Focus and negative polarity in Hindi. Natural Language Semantics 6:57-123.

Lee, Young-Suk, and Laurence Horn. 1994. Any as indefinite plus Even. Ms., Yale University.

Levinson, Stephen. 2000. Presumptive meanings: The theory of Generalized Conversational Implicature. Cambridge, MA: MIT Press.

Ludlow, Peter. 2002. LF and natural logic. In G. Preyer and G. Peter, eds., Logical form and language, 132-168. Oxford: Oxford University Press.

Elena Herburger

Department of Spanish and Portuguese

Georgetown University

ICC 403

Washington, DC 20057

herburge@georgetown.edu

smm23@georgetown.edu 